\title{
Experimental Study on the Role of Chro- matic Dispersion in Continuous-Wave Supercontinuum Generation
}

\author{
Laura Abrardi, Sonia Martín-López, Ana Carrasco-Sanz, Félix Rodríguez-Barrios, Pedro Corredera, \\ Maria Luisa Hernanz, and Miguel González-Herráez
}

\begin{abstract}
The influence of chromatic dispersion on continuous-wave (CW)-pumped supercontinuum (SC) generation in kilometer-long standard fibers is experimentally investigated. We perform our study by means of a tunable, high-power fiber ring laser pumping a dispersion-shifted fiber in the wavelength range of small and medium anomalous dispersion. Our results show that, at low input powers, chromatic dispersion plays a dominant role on nonlinear pump spectral broadening, giving rise to a broader spectrum when pumping just above the zero-dispersion wavelength of the fiber. At higher input powers, however, the width of the generated SC spectrum is mostly due to the Raman effect, hence more independent of the value of the chromatic dispersion coefficient. We show that, in this case, the optimum pumping wavelengths for $\mathrm{SC}$ generation are not so close to the zero-dispersion wavelength of the fiber as in the previous case. In these conditions, as the chromatic dispersion grows, we can obtain square-shaped and high-power density spectra, which seem extremely promising for applications in optical coherence tomography.
\end{abstract}

Index Terms-Chromatic dispersion, dispersive waves, nonlinear optics, soliton self-frequency shift, supercontinuum (SC).

\section{INTRODUCTION}

$\mathbf{N}$ OWADAYS, fiber-based supercontinuum (SC) sources find widespread applications in many different fields, including telecommunications, spectroscopy, and medical imaging techniques like optical coherence tomography [1], [2]. In particular, continuous-wave (CW) SC generation in optical fibers has attracted much attention in recent years for the possibility of developing compact, high-quality sources for ultrahigh resolution optical coherence tomography. Among their good

Manuscript received November 22, 2007; revised June 9, 2008. Current version published February 13, 2009. The authors acknowledge financial support from the Ministerio de Educación y Ciencia through projects TEC2006-09990C02-01 and TEC2006-09990-C02-02, the support from CSIC through project MeDIOMURO, the support from the Comunidad Autonoma de Madrid through the projects FUTURSEN S-0505/AMB/000374 and FACTOTEM S-0505/ESP/ 000417, and the support from Social European Fund through the grant program I3P of CSIC.

L. Abrardi, S. Martín-López, A. Carrasco-Sanz, F. Rodríguez-Barrios, P. Corredera, and M. L. Hernanz and are with the Instituto de Fisica Aplicada, Consejo Superior de Investigaciones Cientificas (CSIC), 28006 Madrid, Spain (e-mail: abrardi@ifa.cetef.csic.es).

M. González-Herráez is with the Departamento de Electronica, Universidad de Alcala. Edificio Politecnico, 28871 Alcala de Henares, Madrid, Spain.

Color versions of one or more of the figures in this paper are available online at http://ieeexplore.ieee.org.

Digital Object Identifier 10.1109/JLT.2008.928211 properties, these sources exhibit extremely short coherence lengths (allowing resolutions of only several micrometers), high power spectral densities [normally in the order of several milliwatts per nanometer $(\mathrm{mW} / \mathrm{nm})]$ and lower values of relative intensity noise (RIN) than their pulsed counterparts [3], [4].

The process of converting a CW laser emission into supercontinuum in optical fibers has been studied intensively in the last years, both experimentally [5]-[10] and theoretically [11]-[14]. These studies have shown that CW SC sources are initiated by modulation instability (MI) of the partially coherent input beam, which can lead to soliton formation and collision processes [14]-[16]. Then, the Raman scattering induces a self-frequency downshift of the generated solitons, so that their final spectral distribution covers a wide wavelength range. The creation of a soliton is accompanied by the generation of a dispersive wave frequency upshifting as the soliton downshifts because of the Raman scattering. Although the dynamics of CW SC generation are reasonably understood nowadays, many efforts are still being done in order to improve and optimize the supercontinuum properties, such as higher spectral power density, larger spectral width, smoother spectral profile, and better long-term stability. As an example, in a previous paper [17], we studied the influence of pump incoherence in CW SC generation. We showed experimentally, that, for a given input power and chromatic dispersion coefficient, there is an optimum value of pump incoherence that yields the most efficient spectral broadening. In this paper, we use a similar methodology to study the dependence of CW SC generation on chromatic dispersion. This study seeks to understand possible strategies to optimize the fiber dispersion so as to improve the SC properties.

Chromatic dispersion plays a key role in the processes of modulation instability and soliton formation. The most obvious limit is that these processes efficiently occur in the anomalous dispersion region of the fiber. Besides, at smaller anomalous dispersion values the MI oscillations are temporally faster and induce shorter pulse formation [18]. This effect leads to the generation of shorter solitons which undergo a larger red-shift per fiber length due to intrapulse Raman scattering [19], [20]. Soliton compression can also be induced by use of dispersion management [9], [10], [21], leading to enhanced supercontinuum spectral width.

In this paper, we present an exhaustive experimental study of the influence of chromatic dispersion on supercontinuum spec- 
tral broadening generated with continuous-wave light in a standard dispersion-shifted fiber (DSF). This is done by tuning the wavelength of the high-power pump laser over the spectral region of small and moderate anomalous chromatic dispersion of the fiber. This paper shows that beyond a certain power level there is a range of chromatic dispersion values for which the spectral width of the supercontinuum remains constant. This range of values is broader as the pump power increases. In addition, we show that the optimum shape of the SC is actually obtained for moderate values of chromatic dispersion. These results seem to be very interesting from a practical and engineering point of view, and we explain them qualitatively based on the established explanation of CW SC generation.

This paper is organized as follows. In Section II, the experimental setup is described. In Section III, we discuss the effect of chromatic dispersion on soliton dynamics and dispersive wave generation; in Section IV, we show experimental results on the dependence of supercontinuum spectral width on chromatic dispersion. Experimental results show that broadband SC can be efficiently generated by $\mathrm{CW}$ pumping over a wide range of dispersion values when using kilometer-long standard fibers and input powers of several watts. Finally, in Section V we derive the conclusions of our study.

\section{EXPERIMENTAL SETUP}

In order to make an experimental study on the influence of chromatic dispersion on nonlinear spectral broadening, we need to have a way to pump the fiber at different dispersion values, or equivalently, at different wavelengths. The wavelength range that we treat here is such that the nonlinear coefficient, Raman gain and losses of the fiber do not vary too much, but the dispersion does vary significantly. The pumping is realized by means of a home-made high-power tunable laser source. Our pump is a fiber-ring laser mainly made up of two erbium-doped fiber amplifiers (EDFAs) and a tunable optical grating filter, as shown in Fig. 1. EDFA1 (EDFA-C17) has gain in the wavelength range of 1525-1570 nm. After EDFA1, an optical grating filter is inserted (TB9 provided by JDS Uniphase Corporation, Milpitas), which is used to select the desired frequency out of the EDFA1 emission. It provides a tuning range of $1525-1625 \mathrm{~nm}$ and a spectral width of 0.5 -nm full-width at half-maximum (FWHM). The grating filter is followed by a variable attenuator (provided by Accelink, Wuhan, China), which is used to control the power inserted into EDFA2. In fact, we want to guarantee that EDFA2 is working at the same saturation level at all wavelengths. EDFA2 is a high-power fiber amplifier (provided by Keopsys, Lannion, France) working in the spectral range 1545-1570 nm, whose output power can take values from $200 \mathrm{~mW}$ to $10 \mathrm{~W}$. A highpower isolator (provided by OPNETI, Shenzhen, China) is inserted at the output of EDFA2 to prevent damage in the amplifier by back-reflected light and backward Raman. The isolator losses are approximately $0.5 \mathrm{~dB}$. Only $0.01 \%$ of the power delivered by EDFA2 is recirculated in the cavity. This is done by means of a set of two calibrated $1 / 99$ couplers. We used the remaining port to monitor the output power of the fiber laser. This arrangement allows us to generate a tunable source whose output power takes the same value for each wavelength during our measurements. The described fiber-ring laser is single mode, it presents

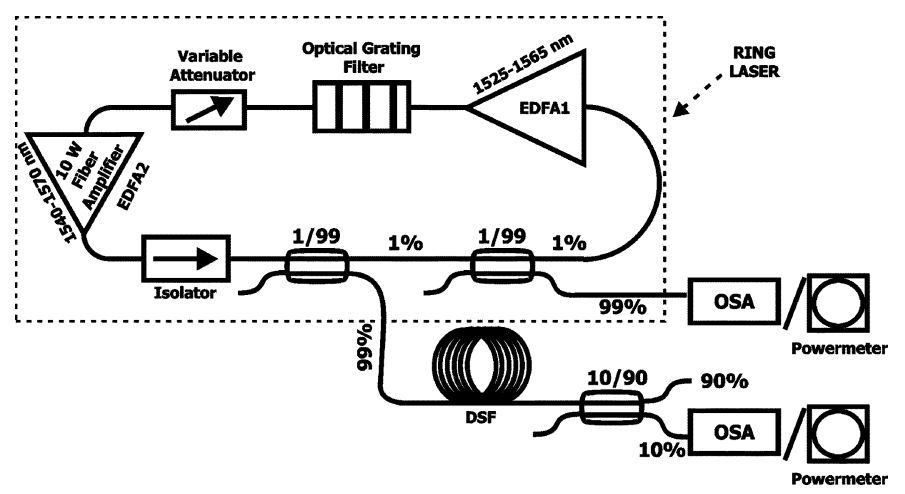

Fig. 1. Experimental setup. EDFA: Erbium-doped-fiber amplifier; DSF: Dispersion-shifted fiber; and OSA: Optical spectrum analyzer.

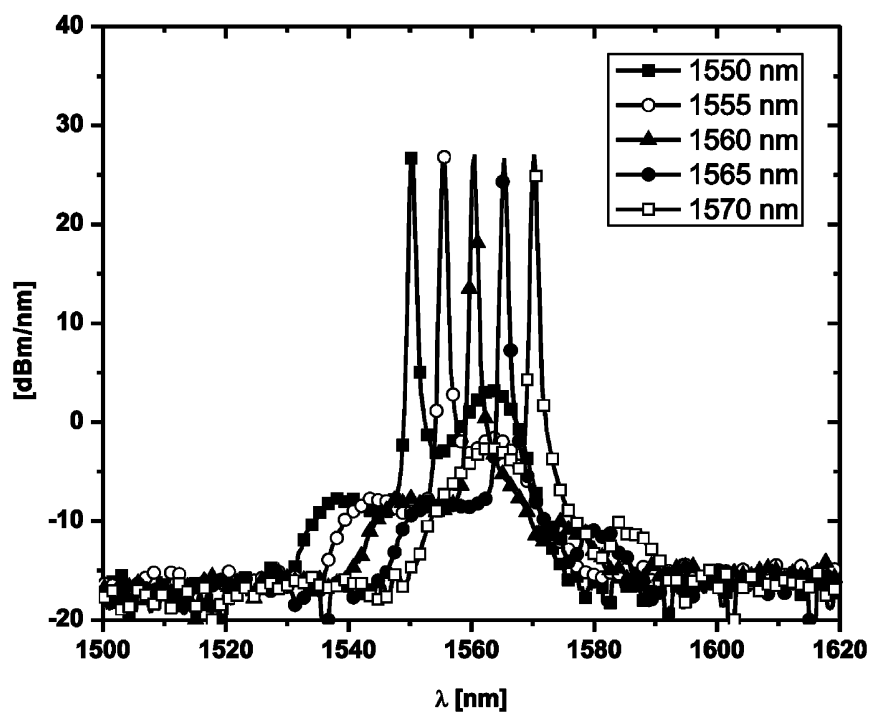

Fig. 2. Laser output at different wavelengths. The total output power is $2 \mathrm{~W}$.

a 0.08 -nm FWHM line width at every wavelength in the available tuning range (i.e., from 1545 to $1570 \mathrm{~nm}$ ) and its power can take values from $500 \mathrm{~mW}$ to $7 \mathrm{~W}$. The laser output can be seen in Fig. 2 at different wavelengths for a total output power of 2 W.

The fiber is an 11-km DSF provided by Corning. The dispersion curve of the fiber has been measured using the phase-shift method described in [22] with a resolution of $2 \mathrm{~nm}$. Both the zero-dispersion wavelength $\lambda_{\mathrm{ZD}}$ and the nonlinear coefficient $\gamma$ have been measured with an alternative scheme using a method based on modulation instability [23]-[25] and found to take values $\lambda_{\mathrm{ZD}}=1553.5 \pm 0.5 \mathrm{~nm}$, and $\gamma=1.7 \mathrm{~W}^{-1} \mathrm{~km}^{-1}$. In the tuning range of our ring-cavity laser, the group-velocity dispersion (GVD) parameter $\beta_{2}$ is linearly decreasing with a slope of $-0.1044 \mathrm{ps}^{2} / \mathrm{km} / \mathrm{nm}$, and it takes value $0.7828 \mathrm{ps}^{2} / \mathrm{km}$ at $\lambda=1545 \mathrm{~nm}$ and $-1.8266 \mathrm{ps}^{2} / \mathrm{km}$ at $\lambda=1570 \mathrm{~nm}$. The thirdand fourth-order-dispersion parameters (TOD and FOD, respectively) can be considered almost constant over the whole tuning range, and they take values $\beta_{3}=0.1358 \pm 0.00377 \mathrm{ps}^{3} / \mathrm{km}$ and $\beta_{4}=-4.8739 \times 10^{-4} \pm 0.0560 \times 10^{-4} \mathrm{ps}^{4} / \mathrm{km}$ respectively. Since higher order dispersion parameters are almost constant, we can make a study of the influence of second-order dispersion parameter on spectral broadening and neglect the influ- 
ence of higher order dispersion terms. The output spectrum is finally analyzed by means of an optical spectrum analyzer (OSA) with a spectral resolution of $1 \mathrm{~nm}$ after passing through a 10/90 coupler, which acts uniquely as fixed attenuator. The input and output power are measured by means of an integrating sphere radiometer whose responsivity is known in the whole tuning range with $1 \%$ uncertainty [26].

\section{ANOMALOUS DisPERSION REGIME AND DISPERSIVE WAVE GENERATION}

This work seeks to give a deeper insight into the effect of chromatic dispersion on supercontinuum generation in kilometer-long conventional fibers. CW-pumped spectral broadening in fibers results from the fission of the partially coherent input CW beam into a sequence of Raman-shifted solitons through the combined effects of modulation instability and stimulated Raman scattering (SRS). CW-induced spectral broadening is initiated by modulation instability which breaks up the $\mathrm{CW}$ radiation into a train of ultrashort pulses when propagating in the wavelength region of anomalous dispersion of the fiber. As the pulses propagate, the MI-generated pulses evolve into fundamental first-order solitons (one-soliton) [12] which undergo a spectral shift towards longer wavelengths due to SRS. This process of soliton self-frequency shift (SSFS) gives rise to a smooth and wide spectrum lying at wavelengths longer than the pump wavelength. In addition, each fundamental soliton can, in the presence of higher-order dispersion, release excess energy in the form of dispersive waves, enhancing the spectral broadening in the normal dispersion region of the fiber [27]. These linear waves are emitted only when the soliton spectrum overlaps with some resonant wave frequencies. The amplitude of the DW radiation is proportional to the amplitude of the soliton at the phase-matched frequencies [27], so that more intense radiation is released by solitons that are closer to $\lambda_{\mathrm{ZD}}$.

The most obvious impact of chromatic dispersion on soliton dynamics appears through MI. In fact, CW-light propagating in the anomalous dispersion regime of an optical fiber breaks up into a train of solitonlike pulses due to MI. As the MI oscillation frequency is inversely proportional to $\sqrt{\beta_{2}}$ [28], shorter solitons are expected to be generated when pumping closer to $\lambda_{\mathrm{ZD}}$ [18], [29]. As shorter solitons red-shift faster due to SSFS [19], [20], a much wider spectrum is expected to be generated when pumping closer to $\lambda_{\mathrm{ZD}}$. Thus, it is clear that under no other effects, the most efficient nonlinear broadening is expected at GVD parameters as close to zero as possible. However, in Section IV, we will see that beyond a certain input power, Raman scattering becomes the dominant nonlinear effect in these kilometer-long fibers. In this case, soliton self-frequency shift is the dominant broadening mechanism, and the importance of chromatic dispersion is radically smaller.

We now look at the blue-shifted part of the SC spectrum. Dispersive waves are not only radiated by fundamental solitons in the presence of higher order dispersion perturbation. If sufficiently high power is injected into the fiber in anomalous dispersion regime, a train of $N$-solitons can be generated. In this case, dispersive waves arise from higher-order soliton, which are unstable under higher-order dispersion and decay into fundamental solitons. These latter ones eject excess of energy as dispersive radiation in the normal dispersion region of the fiber [30]-[34].

Previous theoretical and experimental studies [27], [31], [35] show that dispersive waves are generated when the soliton spectrum overlaps the resonance frequency which fulfills the following phase-matching condition:

$$
\beta\left(\omega_{\mathrm{DW}}\right)-\beta\left(\omega_{\mathrm{S}}\right)=\sum_{n \geq 2}^{M} \frac{\beta_{n}}{n !} \delta \omega^{n}-\frac{\gamma P_{\mathrm{S}}}{2}=0
$$

where $\beta\left(\omega_{\mathrm{DW}}\right)$ is the wave number of the dispersive radiation, $\beta\left(\omega_{\mathrm{S}}\right)$ is the wave number of the soliton, $\delta \omega=\left(\omega_{\mathrm{DW}}-\omega_{\mathrm{S}}\right)$ is the detuning between the radiation frequency $\omega_{\mathrm{DW}}$ and the central soliton frequency $\omega_{\mathrm{S}}$, and $\beta_{n}$ is the $n$th derivative of $\beta(\omega)$ calculated at $\omega_{\mathrm{S}} ; \gamma P_{\mathrm{S}} / 2$ is the soliton nonlinear phase shift, $\gamma$ is the nonlinear parameter of the fiber [28], and $P_{\mathrm{S}}$ is the soliton peak power. This equation allows us to predict the dispersive wave frequency once the dispersion parameters of the fiber and the soliton carrier frequency are known. The resonant radiation frequency depends weakly on the soliton amplitude [27], [36], so that the nonlinear phase-shift term can be often neglected in (1). In this case, the phase matching condition is equivalent to

$$
\sum_{n \geq 2}^{M} \frac{\beta_{n}}{n !} \delta \omega^{n-2}=0
$$

where the two degenerate solutions $\delta \omega=0\left(\omega_{\mathrm{DW}}=\omega_{\mathrm{S}}\right)$ have been obviously discarded. In our experiment, we observed that the blue part of the spectrum shows a maximum in intensity that, for a given input wavelength, always falls at the same frequency for different input powers. As we cannot measure the central frequency of the generated solitons, we make the hypothesis that most of the DWs are radiated at the first stage of soliton formation, when solitons are expected to have central wavelength just above the pump wavelength. According to this hypothesis, we calculated the DW frequency that would be radiated by a soliton at the pump frequency by substituting $\omega_{\mathrm{S}}=\omega_{P}$ in (2) using the series expansion until the fourth order $(M=4)$. We found that the frequency of the maxima of the blue-shifted radiation were in good agreement with this assumption. Measurements were performed at different input powers and are shown in Fig. 3 together with the theoretical curve.

As expected from the phase-matching condition, at higher values of dispersion, the solitons are in resonance with dispersive radiation at larger frequency shifts from the soliton carrier frequency. The experimental data seem to confirm that most radiation is emitted by solitons generated just above the pump wavelength, i.e., at the first stages of soliton formation, before they undergo a significant SSFS. Besides, it seems that these dynamics are not sensitive to the average injected power until a power of several watts ( $P=3 \mathrm{~W}$ in our case) is injected for which blue-shifted components are still amplified, but no maxima can be seen anymore. In Fig. 4, we show the output spectra obtained for input powers of $250 \mathrm{~mW}$ (a) and $1 \mathrm{~W}$ (b). The pump wavelength and the corresponding chromatic dispersion values are reported in the legend. Output spectra 


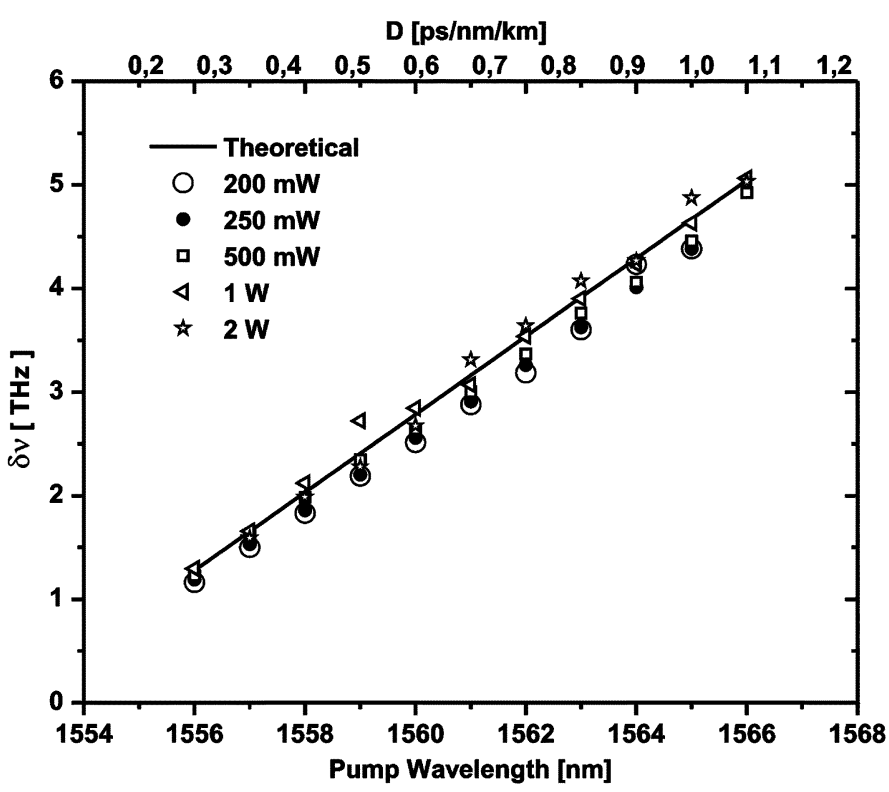

Fig. 3. Frequency shift between the DW and the pump frequency as function of pump wavelength for different input powers.

at $250 \mathrm{~mW}$ clearly show MI sidebands and dispersive waves. At low pump wavelengths $(1556 \mathrm{~nm})$, the spectrum exhibits a typical MI asymmetric profile and intense dispersive waves, while at longer pump wavelengths (i.e., larger dispersion) the MI asymmetry is less evident and lower intensity dispersive waves are generated.

It is worth noting that, for the same input power, the intensity of the dispersive wave decreases as the pump wavelength increases. This is consistent with the fact that the energy transfer from the soliton to the DWs depends on the spectral overlap between these two waves [27], [37]. As they become more apart with increasing pump wavelength, the overlap between them is smaller. Besides, the spectral gap between the DW peak and the residual pump is increasing when pumping at longer wavelengths. In Fig. 4(b), the output spectra are shown for an input power of $1 \mathrm{~W}$. At this input power, MI sidebands cannot be appreciated anymore and large spectral broadening and strong pump depletion start to take place. Dispersive waves present higher intensity then the previous case. This is clearly visible in Fig. 4(b). We also show that, as expected, for a too large detuning between the pump and $\lambda_{\mathrm{ZD}}$, the soliton spectrum does not overlap with the phase-matched frequencies and DW amplification cannot take place, which is the case for example when pumping at $1570 \mathrm{~nm}$. This process, which is consistent with previous results in microstructured fibers [31], [33], is made clearer by a 3-D representation of our spectra. In Fig. 5, the output spectrum evolution is shown as function of pump wavelength for an input power of $1 \mathrm{~W}$. There is a clear sign of dispersive waves, and the transition from normal to anomalous dispersion around $1554 \mathrm{~nm}$ is also evident by a large spreading of the output spectrum. We note that the dependence of MI and dispersive waves on chromatic dispersion gives rise to SC spectra having a completely different shape for different pump wavelengths and powers. In particular, at this value of input power, the spectrum is flatter and broader

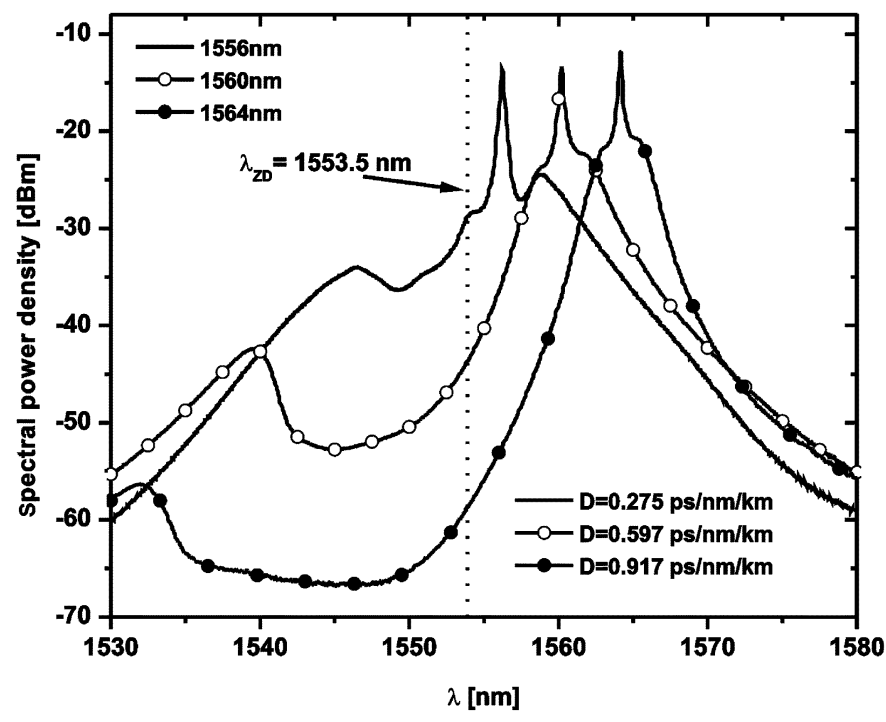

(a)

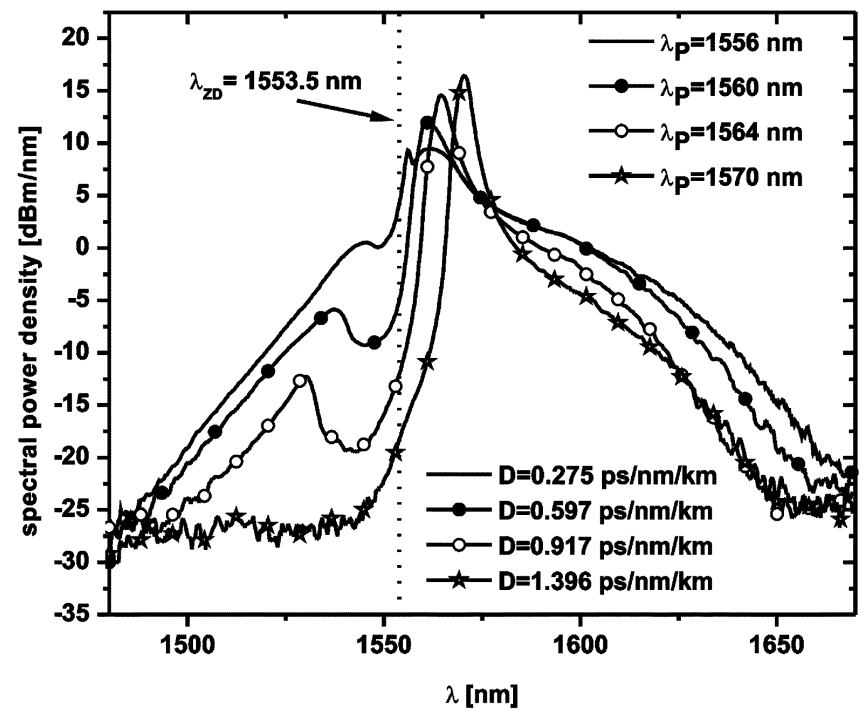

(b)

Fig. 4. Modulation instability and dispersive wave generation in different dispersion regimes. (a) Spectra are shown for an input power of $250 \mathrm{~mW}$ and (b) spectra are shown for an input power of $1 \mathrm{~W}$.

when the pump wavelength is just above the zero-dispersion wavelength of the fiber $\lambda_{\mathrm{ZD}}=1553.5 \mathrm{~nm}$. In Fig. 7, the ratio between the maximum DW intensity and the residual pump intensity is plotted for different average input powers. It is interesting to observe that this ratio grows with increasing input power below $P=1 \mathrm{~W}$. When higher powers are injected into the fiber, this ratio remains almost constant. Moreover, when the pump power is higher than $1 \mathrm{~W}$, dispersive waves start to undergo attenuation with increasing input power, as it can be seen in Fig. 6. In this case, however, flatter supercontinua are obtained for longer pump wavelengths (i.e., larger values of dispersion), as we will see in Section IV. Since the Raman threshold in our case is estimated to be $P_{c r} \sim 3 \mathrm{~W}$ [28] by taking the Raman gain coefficient $g_{R}=6.3 \times 10^{-12} \mathrm{~cm} / \mathrm{W}$ for silica fibers at $1550 \mathrm{~nm}$ [20], [38], we believe that these results are the clear signature of the increasing role of Raman 


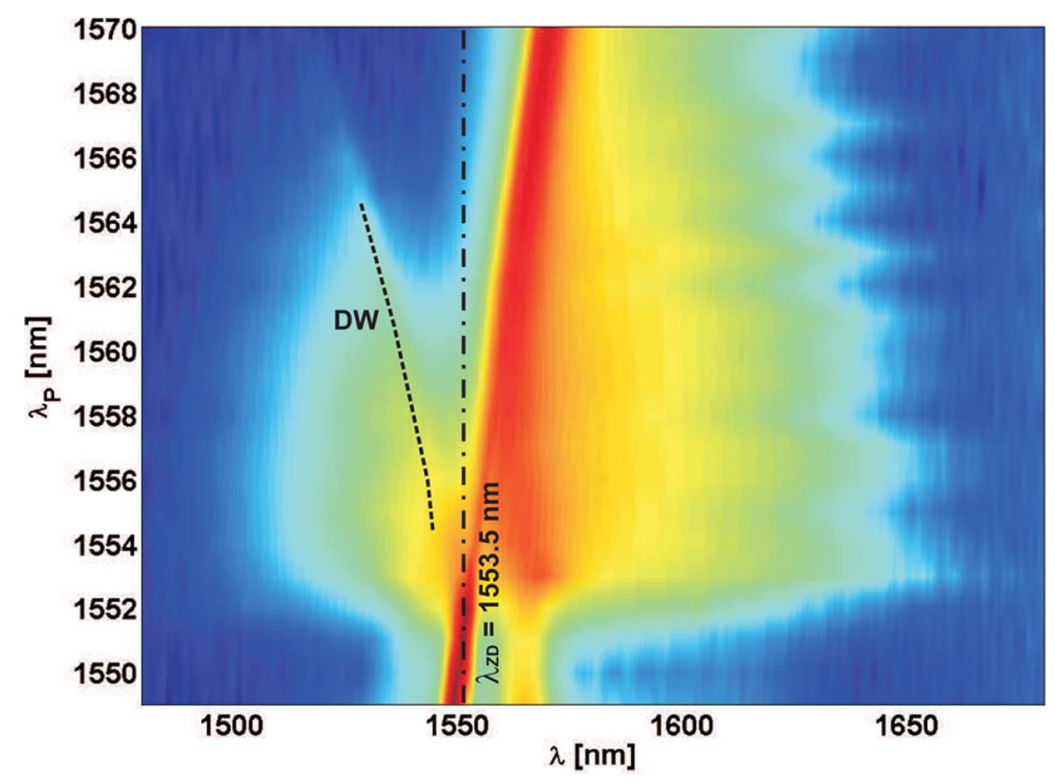

Fig. 5. Output spectra as function of pump wavelength $\lambda_{P}$ (vertical axis) for an input power of $1 \mathrm{~W}$. We can see that DWs give a significant contribution to spectral broadening only when pumping just above $\lambda_{\mathrm{ZD}}$. For increasing pumping wavelength, their intensity is decreasing and the spectral gap between the residual pump and the DW peak is increasing. For $\lambda_{P}>1566 \mathrm{~nm}$, there is no DW amplification and the whole SC spectrum lies above $\lambda_{\mathrm{ZD}}$.

scattering on SC dynamics in this kilometer-long silica fiber. When increasing the input power, shorter solitons are expected to form and, since they red-shift faster due to SSFS, lower DW amplification takes place. It is now important to point out that the presence of a well-defined peak in the blue part of the spectrum seems to indicate that higher order solitons are not generated or, eventually, only low-order solitons are formed $(N \sim 1)$. In fact, if the dispersive radiation were the result of soliton fission dynamics, a much broader and flatter blue-shifted spectrum would be generated due to the contribution of the $N$-soliton decay into $N$ fundamental solitons of different central frequency [30], [31]. Besides, when DWs are radiated by fundamental solitons from higher-order soliton fission, a maximum of intensity should appear at the blue edge of the spectrum [31], [33], which is not our case. Instead, our results are consistent with theoretical predictions for which in $\mathrm{CW}$-pumping regimes fundamental solitons of slightly different amplitude and time duration originate from phase noise of the pump due to MI [11], [12], [14]. Immediately after formation, they release nonsolitonic radiation in the presence of higher order dispersion and continue to radiate some energy while Raman red-shifting until stability is reached. On the other side, some authors [39] also proved that MI-induced solitons have too long a time duration to undergo a significant SSFS for broad supercontinua to be generated. Some new effects other than soliton fission have to be taken into account. For this reason, our results seem to be in very good agreement with the latest theoretical studies that attribute to soliton collisions a significant role in enhancing the spectral width by leading to shorter soliton formation [14], [16]. Moreover, new results have been published that reveal the influence of statistical processes on enhanced spectral broadening because of the generation of rare extremely red-shifted solitons [40], [41]. In particular, our experimental conditions well correspond to the theoretical model presented by Dudley et al. [41] where the authors point

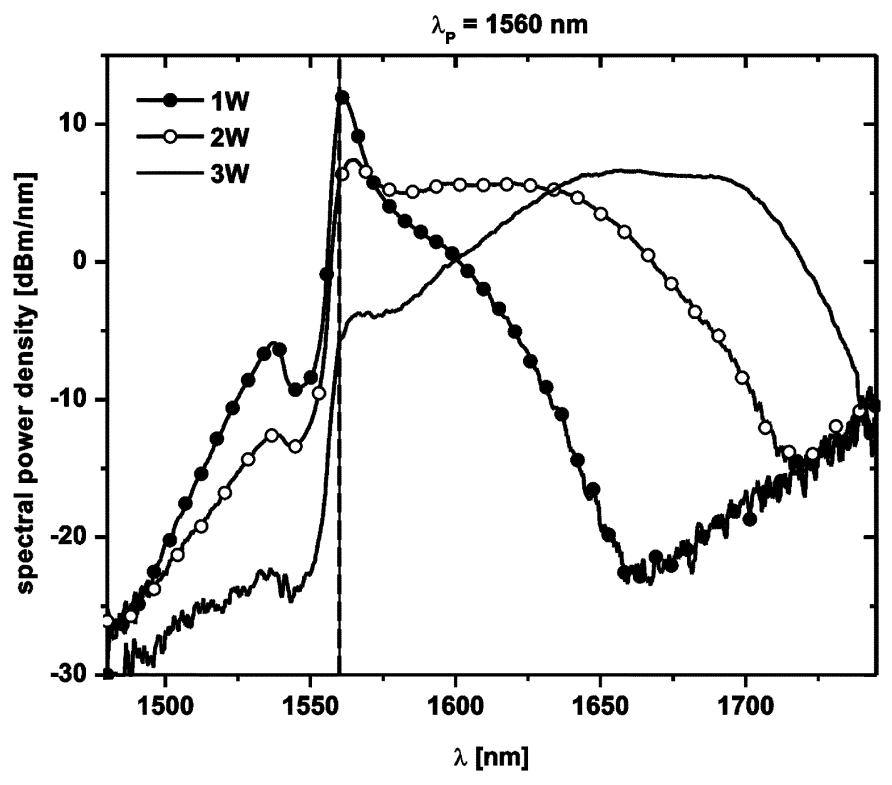

Fig. 6. Supercontinuum spectra obtained at three different input powers. The pump wavelength is $\lambda_{P}=1560 \mathrm{~nm}$ and marked with a dashed line in the figure.

out the role of pump noise as well as of the interplay between MI and Raman scattering on rogue soliton generation, that leads to further spectral broadening at longer wavelengths.

\section{INFLUENCE OF CHROMATIC DISPERSION ON SC SPECTRAL BROADENING}

In this section, we analyze experimentally how chromatic dispersion affects SC spectral broadening in high-power regimes when propagating a $\mathrm{CW}$ radiation into a kilometer-long conventional DSF. As we have already mentioned, our high-power tunable CW laser allows us to pump at different dispersion values 


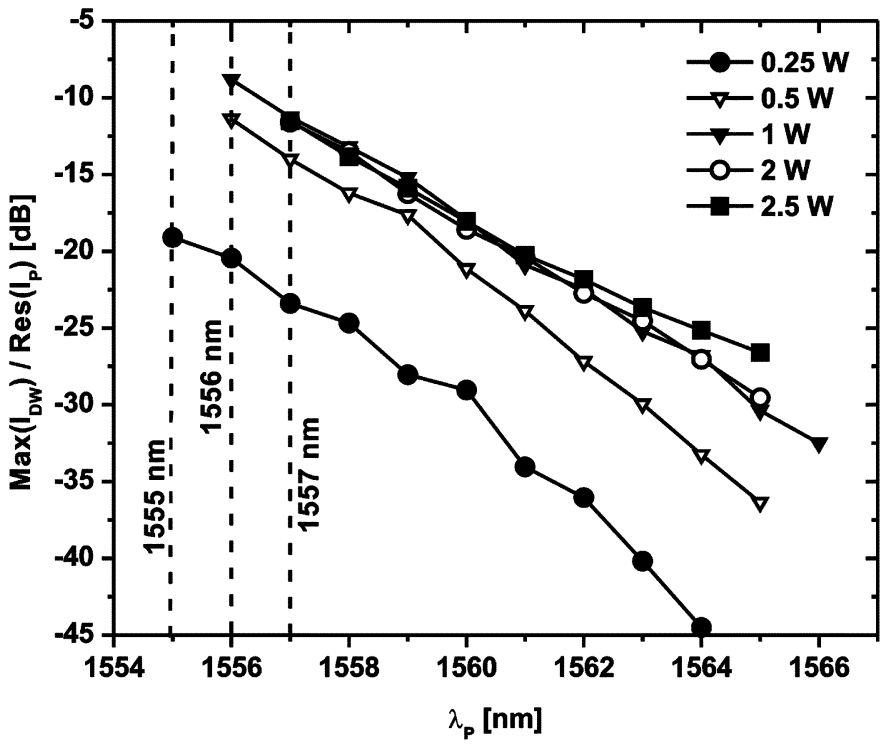

Fig. 7. Ratio between the DW maximum intensity and the residual pump intensity as function of pump wavelength for different input powers. The relative intensity of DWs is increasing only for pump powers below the Raman threshold $P=1 \mathrm{~W}$.

of the fiber. Now we can study how chromatic dispersion influences the properties of SC spectra at different values of the total injected power. As it has been already pointed out in a recent SC review [42], these results are of very practical interest because there is a lack of experimental studies in the field of $\mathrm{CW}$ $\mathrm{SC}$ generation due to the limited high-power CW sources available. In this section, we want to analyze the SC spectral width. As we have already mentioned, SC spectra exhibit very different shapes when pumping at different wavelengths [see Fig. 4(b)]. In order to compare the mentioned spectra among each other, we need to introduce a clear definition of SC spectral width which takes into account their different shape and their different spectral power density distribution. So far, SC spectral width has been usually defined in logarithmic scale as the width measured $20 \mathrm{~dB}$ down from the highest peak of the spectrum. This definition hardly takes into account the effective available spectral width when the spectrum takes an asymmetric and complicated shape or a significant gap between its longer-wavelength part and its blue-shifted part. This situation makes difficult to compare spectral widths obtained for the same input power but at different pump wavelengths by using the common definition of spectral width. In order to overcome this problem we decided to use another definition of spectral width according to the standards we found in literature. In ultrashort pulse measurements, a definition of equivalent pulse width (EPW) is used when the pulse shape is quite complicated (see [43]). Following this idea, we adopted the definition of equivalent spectral width (ESW) to assign a spectral width value to each of our spectra with a consistent and unique method. We define the ESW of a SC source as

$$
w_{e}=\frac{2}{I_{\max }} \int_{-\infty}^{\infty} I(\lambda) d \lambda
$$

where $w_{e}$ is the $\mathrm{ESW}, I(\lambda)$ is the spectral power density in $\mathrm{mW} / \mathrm{nm}$, and $I_{\max }$ is the maximum power density of the spectrum in $\mathrm{mW} / \mathrm{nm}$. An illustration of the definition of ESW can be seen in Fig. 8. It is worth noticing that this definition uses the total power under the spectral curve, so that all the generated spectral components are taken into account. Moreover, a higher value of ESW is assigned to flatter spectra because the maximum peak is usually lower. For example, when full depletion occurs, the maximum spectral power density usually takes a lower value, generating a higher value of ESW. This definition is very useful when spectral widths of different supercontinua have to be compared. In fact, it favors wider and flatter profiles because it assigns them a higher ESW value. By using the definition of ESW, we want to study the influence of chromatic dispersion on SC spectral broadening. With the help of our tunable source, we can pump in the spectral range from 1549 to 1570 $\mathrm{nm}$, where the chromatic dispersion of the fiber takes values from $-0.291 \mathrm{ps} / \mathrm{nm} / \mathrm{km}$ to $+1.396 \mathrm{ps} / \mathrm{nm} / \mathrm{km}$. Within this spectral range, all the rest of the higher order dispersion terms, the nonlinear coefficient and the attenuation of the fiber remain reasonably constant.

The ESW as function of pump wavelength is shown in Fig. 9 for different input powers. As it can be seen, for input powers below the Raman threshold $\left(P_{c r} \sim 3 \mathrm{~W}\right)$, there is an optimum pump wavelength for which the ESW is maximum. This demonstrates that, in this range of powers, the optimal pump wavelength to generate a SC source falls just above the $\lambda_{\mathrm{ZD}}$ of the fiber, which is in good agreement with the discussion of Section III. At an input power of 0.5 and $1 \mathrm{~W}$, this maximum corresponds to the pump wavelength of 1554 $\mathrm{nm}$, which is just above the $\lambda_{\mathrm{ZD}}$ of the fiber. In fact, the output spectra show a quite different shape and width when pumping at different wavelengths with a total power, as we have seen in Fig. 4(b). The closer to the $\lambda_{\mathrm{ZD}}$ the laser wavelength is, the broader and flatter the output spectrum is, at expense of the residual pump power. In all cases, the transition from normal to anomalous dispersion is evident by the presence of a steep increase in the spectral broadening around the $\lambda_{\mathrm{ZD}}$ of the fiber. At higher input powers, the behavior changes. At an input power of $2 \mathrm{~W}$, there is an optimal pump wavelength located at $1557 \mathrm{~nm}$, where the ESW is maximum, but a quite wide spectral broadening can be obtained also at other pump wavelengths around this value. Besides, the maximum ESW at this input power is higher than for 2.5 and $3 \mathrm{~W}$. This is due to the fact that at this power, output spectra show a quite uniform and squared shape, and a relatively small maximum, as it can be seen in Fig. 10(a) but the mechanisms leading to this behavior are not yet clear. This gives rise to a higher ESW in a consistent way with the definition of ESW which favors smoother and flatter profiles. We can also observe that when pumping just above $\lambda_{\mathrm{ZD}}$ [spectrum at $\lambda_{P}=1554 \mathrm{~nm}$ in Fig. 10(a)], nonsolitonic radiation is clearly visible but it does not present any local maximum. We believe this is due to the interference between solitons and the DWs that gives rise to the generation of new components at frequencies between the solitons and the dispersive waves, as it was already observed in [32]. When powers higher than $2 \mathrm{~W}$ are injected into the fiber, the same value of ESW can be obtained when pumping over a 


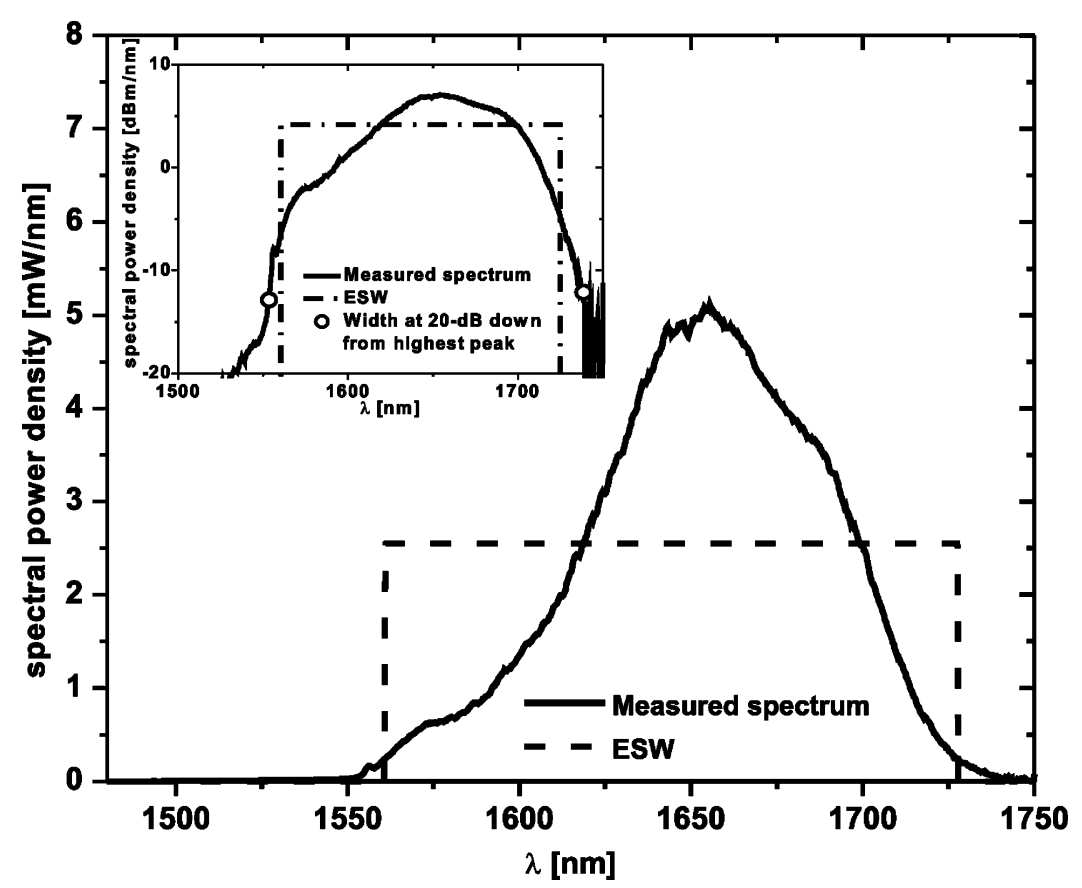

Fig. 8. Equivalent spectral width. The peak of the equivalent spectrum is equal to half the peak of the real spectrum. The width of the equivalent spectrum is such that its area is equal to that of the real one. The equivalent spectrum is centered at such a wavelength so as to have the minimum mean-square error between the real spectrum and the equivalent one. The calculated ESW is compared with the standard measurement $20 \mathrm{~dB}$ down from the highest peak in logarithmic scale.

large spectral region. In Fig. 10(b), we can see the output spectra obtained at different pumping wavelengths for an input power of $3 \mathrm{~W}$. The behavior changes dramatically. First of all, pump depletion occurs also at longer pumping wavelengths. Second, flat and square-shaped profiles are obtained also at longer pumping wavelengths. Third, dispersive waves do not present a maximum at any pump wavelength and they appear more attenuated than for lower input powers. This latter effect, which can be clearly seen in Fig. 6, is probably due to an increasing influence of Raman scattering. In fact, we clearly observe an increasing attenuation of the blue-shifted part of the spectrum and a further amplification of the longer-wavelength components with increasing input power. This effect must be taken into account because it strongly limits the performances of supercontinua in conventional fibers. In fact, although long propagation lengths allow to compensate for a smaller nonlinearity compared to highly nonlinear fibers (as the SSFS is proportional to the propagation distance [20]), broad and high-power spectra can only be obtained at wavelengths above $\lambda_{\mathrm{ZD}}$. This dynamics limit the spectral region of available SC sources in conventional fibers. In order to prove this, we give a rough estimation of the contribution of DWs to the total SC spectrum by calculating the ratio between the spectral power at shorter wavelengths than $\lambda_{\mathrm{ZD}}$ and the total spectral power. It is shown in Fig. 11 for different input powers and wavelengths. It is clear that the contribution of the DWs to spectral broadening is decreasing for increasing input powers and for larger values of dispersion. Dispersive waves tend to disappear with increasing power and an efficient Raman SSFS gives rise to a further red-shifted broad and smooth spectrum. The central wavelength (the average wavelength weighted over the spectral distribution) for three different input powers has been plotted in Fig. 12. As it can

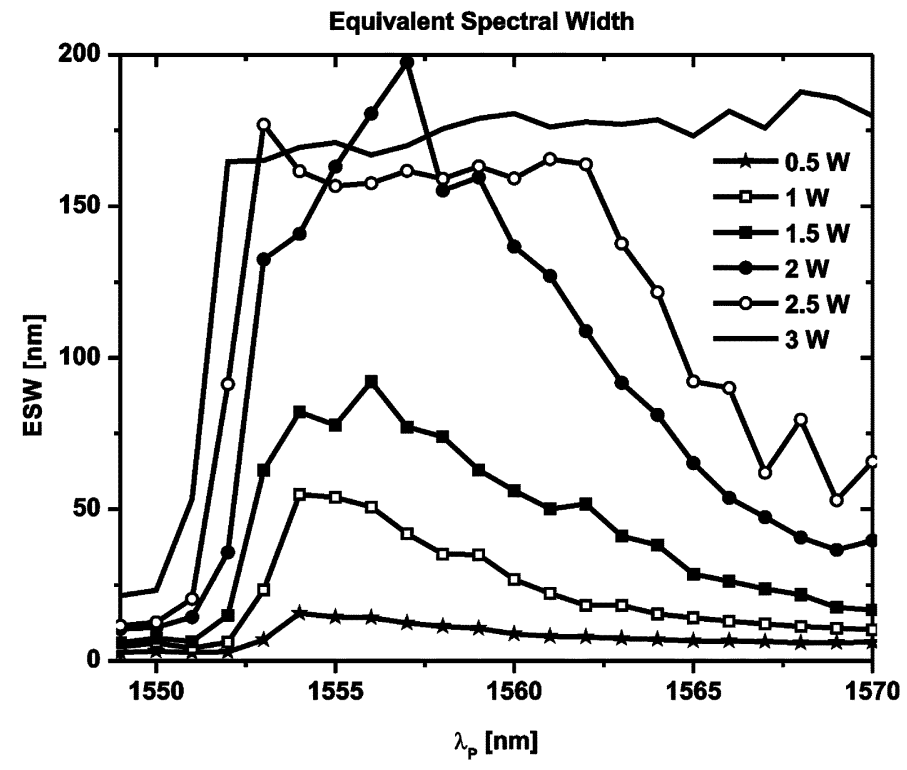

Fig. 9. Supercontinuum spectral broadening at different input powers as function of pump wavelength.

be seen, it does not change significantly with the input laser wavelength but it is significantly red-shifted with increasing input power.

\section{CONCLUSION}

We have studied experimentally the influence of chromatic dispersion on nonlinear pump spectral broadening and $\mathrm{CW}$-pumped supercontinuum generation in kilometer-long standard fibers. This study has been done by pumping a dispersion-shifted fiber with a high-power tunable CW laser source. 


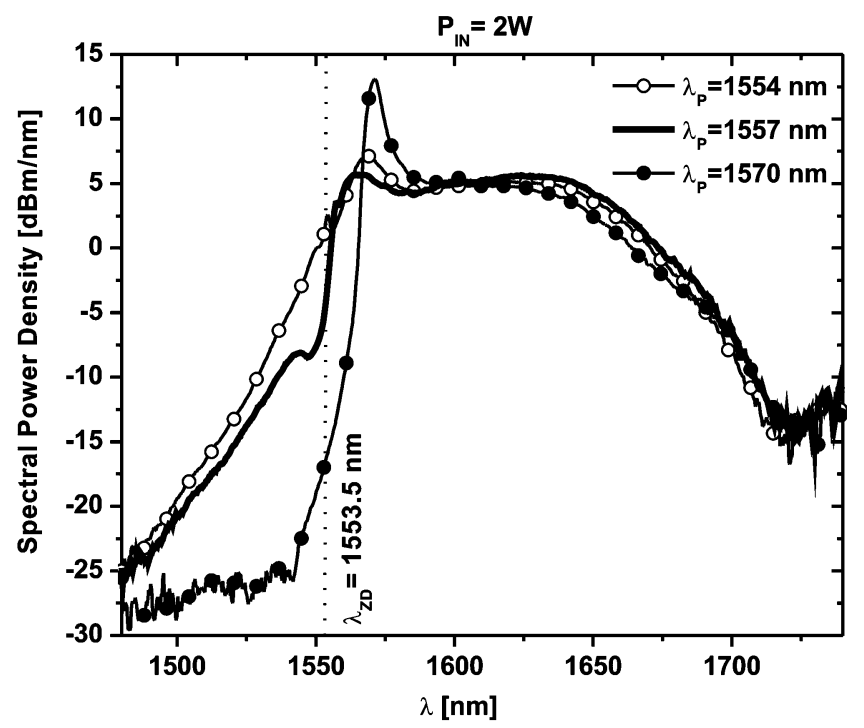

(a)

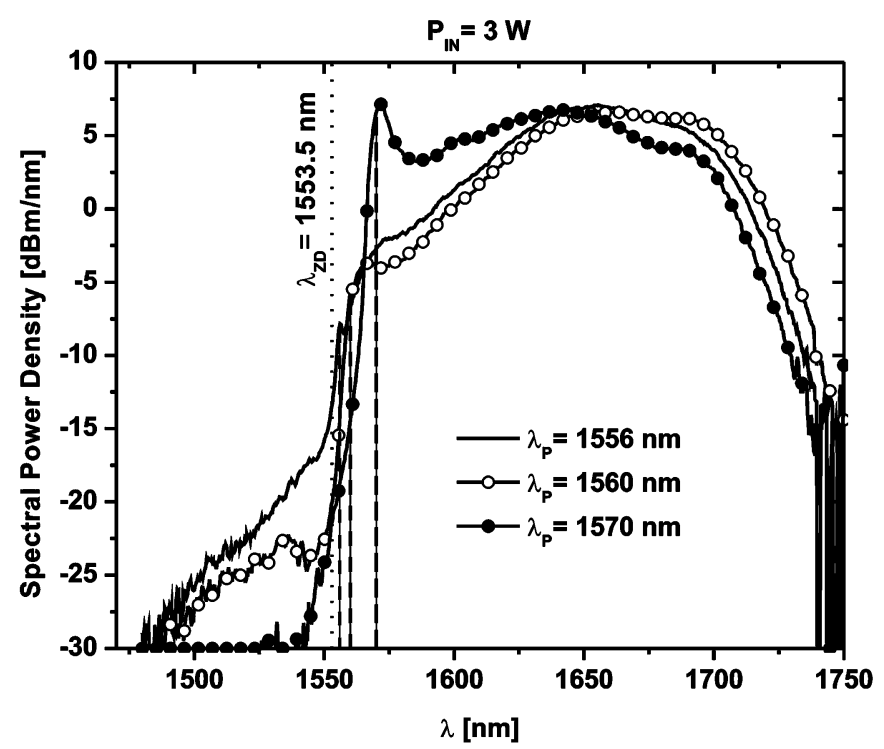

(b)

Fig. 10. Supercontinuum spectra obtained at three different pump wavelengths. (a) Three output spectra are shown for an input power of $2 \mathrm{~W}$. We can see that the spectrum at $\lambda_{P}=1557 \mathrm{~nm}$ presents a lower maximum than the other two spectra and this gives rise to a higher value of ESW. (b) Three output spectra are shown for an input power of $3 \mathrm{~W}$. We can see that there is an amplification of the longer-wavelength components at expenses of the shorter-wavelength components for pump wavelengths just above $\lambda_{\mathrm{ZD}}$. At longer wavelengths $\left(\lambda_{P}=1570 \mathrm{~nm}\right)$, a broad and square-shaped spectrum is obtained.

By sweeping the wavelength of the laser over a small range, we can propagate the pump under very different values of chromatic dispersion but similar values of nonlinear coefficient, attenuation, and higher-order dispersive terms. For a given fiber length, our results show that, at low input powers, chromatic dispersion plays a dominant role on nonlinear pump spectral broadening, giving rise to a broader spectrum when pumping just above the zero-dispersion wavelength of the fiber. When the input power approaches the Raman threshold, however, the width of the generated supercontinuum spectrum is mostly due to the Raman effect, hence more independent of the value of the chromatic dispersion coefficient. We also show that, in this

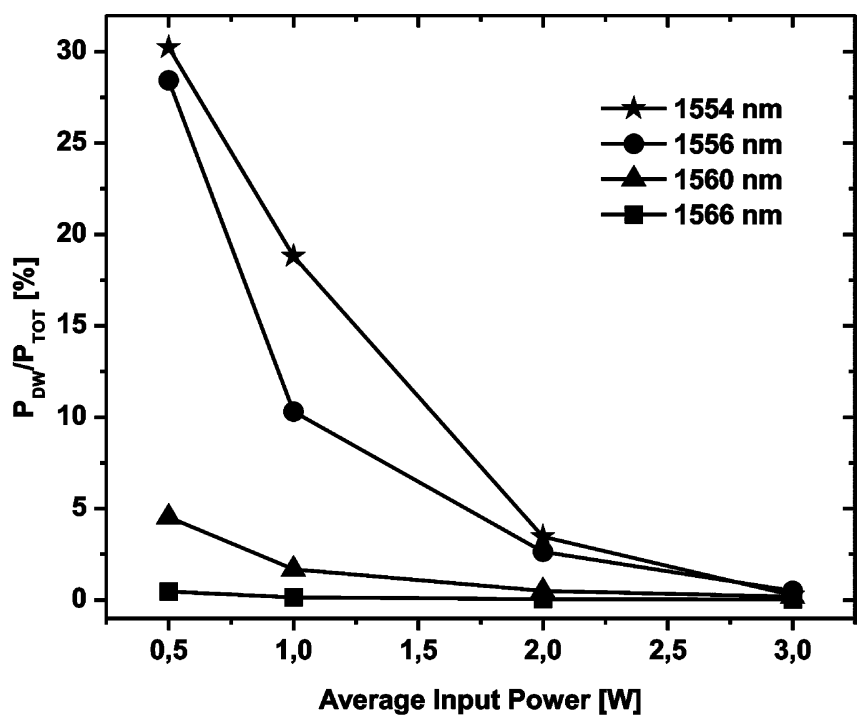

Fig. 11. This shows the contribution of the DWs to the total spectral broadening. This is done by calculating the ratio between the spectral intensity generated in the anomalous dispersion region of the fiber and the intensity of the whole SC spectrum. Values are shown for different pump wavelengths.

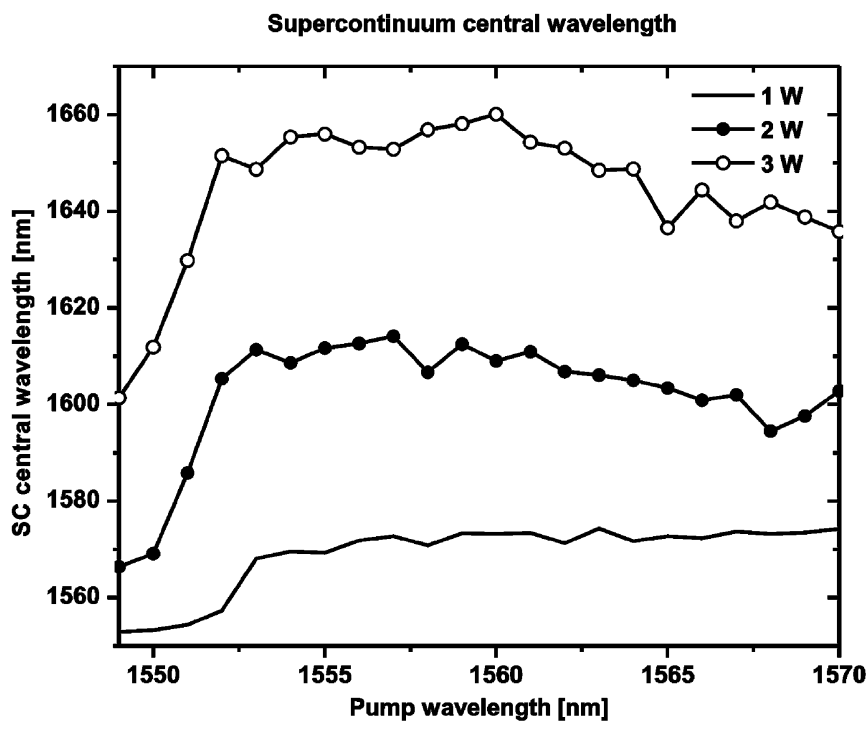

Fig. 12. Supercontinuum central wavelength as function of pump wavelength. Values are shown for input powers of 1,2 , and $3 \mathrm{~W}$.

case, the optimum pumping wavelengths for supercontinuum generation are not so close to the zero-dispersion wavelength of the fiber as in the previous case. We believe that these ideas will be worthy for the engineering of CW-pumped SC sources.

\section{ACKNOWLEDGMENT}

The authors would like to thank Prof. López-Higuera from the University of Cantabria, Santander, for generously lending us his optical filter and Dr. Ania-Castañón from Institute of Optics "Daza de Valdés" (CSIC), Madrid, for useful discussions.

\section{REFERENCES}

[1] J. G. Fujimoto, "Optical coherence tomography for ultra high resolution in vivo imaging," Nat. Biotechnol., vol. 21, no. 11, pp. 1361-1367, 2003. 
[2] P. L. Hsiung, Y. Chen, T. H. Ko, J. G. Fujimoto, C. J. S. de Matos, S. V. Popov, J. R. Taylor, and V. P. Gapontsev, "Optical coherence tomography using a continuous-wave, high power, Raman continuum light source," Opt. Express, vol. 12, no. 22, pp. 5287-5295, 2004.

[3] C. J. S. D. Matos, S. V. Popov, and J. R. Taylor, "Temporal and noise characteristics of continuous-wave-pumped continuum generation in holey fibers around 1300 nm," Appl. Phys. Lett., vol. 85, no. 14, pp. 2706-2708, 2004

[4] S. Martin-Lopez, M. Gonzalez-Herraez, A. Carrasco-Sanz, F. Vanholsbeeck, S. Coen, H. Fernandez, J. Solis, P. Corredera, and M. L. Hernanz, "Broadband spectrally flat and high power density light source for fibre sensing purposes," Meas. Sci. Technol., vol. 17, no. 5, pp. 1014-1019, 2006.

[5] M. Prabhu, A. Taniguhci, S. Hirose, J. Lu, M. Musha, A. Shirakawa, and K. Ueda, "Supercontinuum generation using Raman fiber laser," Appl. Phys. B-Lasers O., vol. 77, no. 2-3, pp. 205-210, 2003.

[6] M. Gonzalez-Herraez, S. Martin-Lopez, P. Corredera, M. L. Hernanz, and P. R. Horche, "Supercontinuum generation using a continuouswave Raman fiber laser," Opt. Commun., vol. 226, no. 1, pp. 323-328, 2003.

[7] P. A. Champert, V. Couderc, and A. Barthelemy, "1.5-2.0 $\mu \mathrm{m}$ multiwatt continuum generation in dispersion-shifted fiber by use of highpower continuous-wave fiber source," IEEE Photon. Technol. Lett., vol. 16, no. 11, pp. 2445-2447, 2004.

[8] A. K. Abeeluck, C. Headley, and C. G. Jorgensen, "High-power supercontinuum generation in highly nonlinear, dispersion-shifted fibers by use of a continuous-wave Raman fiber laser," Opt. Lett., vol. 29, no. 18, pp. 2163-2165, 2004

[9] T. Sylvestre, A. Vedadi, H. Maillotte, F. Vanholsbeeck, and S. Coen, "Supercontinuum generation using continuous-wave multiwavelength pumping and dispersion management," Opt. Lett., vol. 31, no. 13, pp. 2036-2038, 2006.

[10] L. Abrardi, S. Martin-Lopez, A. Carrasco-Sanz, P. Corredera, M. L. Hernanz, and M. Gonzalez-Herraez, "Optimized all-fiber supercontinuum source at $1.3 \mu \mathrm{m}$ generated in a stepwise dispersion-decreasingfiber arrangement," IEEE Photon. Technol. Lett., vol. 25, no. 8, pp. 2098-2012, 2007.

[11] A. Mussot, E. Lantz, H. Maillotte, T. Sylvestre, C. Finot, and S. Pitois, "Spectral broadening of a partially coherent $\mathrm{CW}$ laser beam in singlemode optical fibers," Opt. Express, vol. 12, no. 13, pp. 2838-2843, 2004.

[12] F. Vanholsbeeck, S. Martin-Lopez, M. Gonzalez-Herraez, and S. Coen, "The role of pump incoherence in continuous-wave supercontinuum generation," Opt. Express, vol. 13, no. 17, pp. 6615-6625, 2005.

[13] S. M. Kobtsev and S. V. Smirnov, "Modelling of high-power supercontinuum generation in highly nonlinear dispersion shifted fibers at $\mathrm{CW}$ pump," Opt. Express, vol. 13, no. 18, pp. 6912-6918, 2005.

[14] M. H. Frosz, O. Bang, and A. Bjarklev, "Soliton collision and Raman gain regimes in continuous-wave pumped supercontinuum generation," Opt. Express, vol. 14, no. 20, pp. 9391-9407, 2006.

[15] M. N. Islam, G. Sucha, I. Bar-Joseph, M. Wegener, J. P. Gordon, and D. S. Chemla, "Femtosecond distributed soliton spectrum in fibers," $J$. Opt. Soc. Amer. B, vol. 6, no. 6, pp. 1149-1158, 1989.

[16] N. Korneev, E. A. Kuzin, B. Ibarra-Escamilla, M. Bello-Jiḿnez, and A. Flores-Rosas, "Initial development of supercontinuum in fibers with anomalous dispersion pumped by nanosecond-long pulses," Opt. Express, vol. 16, no. 4, pp. 2636-2645, 2008.

[17] S. Martin-Lopez, M. Gonzalez-Herraez, P. Corredera, M. L. Hernanz, and A. Carrasco, "Experimental investigation of the effect of pump incoherence on nonlinear pump spectral broadening and continuous-wave supercontinuum generation," Opt. Lett., vol. 31, no. 23, pp. $3477-3479,2006$.

[18] A. Hasegawa, "Generation of a train of soliton pulses by induced modulation instability in optical fibers," Opt. Lett., vol. 9, no. 7, pp. 288-290, 1984.

[19] F. M. Mitschke and L. F. Mollenauer, "Discovery of the soliton selffrequency shift," Opt. Lett., vol. 11, no. 10, pp. 659-661, 1986.

[20] J. P. Gordon, "Theory of soliton self-frequency shift," Opt. Lett., vol. 11, no. 10, pp. 662-664, 1986.

[21] J. N. Kutz, C. Lynga, and B. J. Eggleton, "Enhanced supercontinuum generation through dispersion-management," Opt. Express, vol. 13, no. 11, pp. 3989-3998, 2005.

[22] B. Costa, D. Mazzoni, M. Puleo, and E. Vezzoni, "Phase-shift technique for measurement of chromatic dispersion in optical fibers using LEDs," IEEE J. Quantum Electron., vol. 18, no. 10, pp. 1509-1515, 1982.
[23] M. Artiglia, E. Ciaramella, and B. Sordo, "Using modulation instability to determine Kerr coefficient in optical fibers," Electron. Lett., vol. 31, no. 12, pp. 1012-1013, 1995.

[24] C. Mazzali, D. F. Grosz, and H. L. Fragnito, "Simple method for measuring dispersion and nonlinear coefficient near the zero-dispersion wavelength of optical fibers," IEEE Photon. Technol. Lett., vol. 11, no. 2, pp. 251-253, 1999.

[25] J. Fatome, S. Pitois, and G. Millot, "Measurement of nonlinear and chromatic dispersion parameters of optical fibers using modulation instability," Opt. Fiber Technol., vol. 12, no. 3, pp. 243-250, 2006.

[26] A. Carrasco-Sanz, F. Rodriguez-Barrios, P. Corredera, S. MartinLopez, M. Gonzalez-Herraez, and M. L. Hernanz, "An integrating sphere radiometer as a solution for high power calibrations in fiber optics," Metrologia, vol. 43, no. 2, pp. 145-150, 2006.

[27] N. Akhmediev and M. Karlsson, "Cherenkov radiation emitted by solitons in optical fibers," Phys. Rev. A, vol. 51, no. 3, pp. 2602-2607, 1995.

[28] G. P. Agrawal, Nonlinear Fiber Optics. New York: Academic, 2001.

[29] K. Tai, A. Tomita, J. L. Jewell, and A. Hasegawa, "Generation of subpicosecond solitonlike optical pulses at $0.3 \mathrm{THz}$ repetition rate by induced modulation instability," Appl. Phys. Lett., vol. 49, no. 5, pp. 236-238, 1986.

[30] Y. Kodama and A. Hasegawa, "Nonlinear pulse propagation in a monomode dielectric guide," IEEE J. Quantum Electron., vol. 23, no. 5, pp. 510-524, 1987.

[31] A. V. Husakou and J. Hermann, "Supercontinuum generation, fourwave mixing, and fission of higher-order solitons in photonic crystal fibers," J. Opt. Soc. Amer. B, vol. 19, no. 9, pp. 2171-2182, 2002.

[32] J. Hermann, U. Griebner, N. Zhavoronkov, A. Husakou, D. Nickel, J. C. Knight, W. J. Wadsworth, P. S. J. Russel, and G. Korn, "Experimental evidence for supercontinuum generation by fission of higherorder solitons in photonic fibers," Phys. Rev. Lett., vol. 88, no. 17, pp. 173901.1-173901.4, 2002.

[33] G. Genty, M. Lehtonen, and H. L. M. Kaivola, "Enhanced bandwidth of supercontinuum generated in microstructured fibers," Opt. Express, vol. 12, no. 15, pp. 3471-3480, 2004.

[34] M. H. Frosz, P. Falk, and O. Bang, "The role of the second zero-dispersion wavelength in generation of supercontinua and bright-bright soliton-pairs across the zero-dispersion wavelength," Opt. Express, vol. 13, no. 16, pp. 6181-6192, 2005.

[35] K. M. Hilligsøe, H. N. Paulsen, J. Thøgersen, S. R. Keiding, and J. J. Larsen, "Initial steps of supercontinuum generation in photonic crystal fibers," J. Opt. Soc. Amer. B, vol. 20, no. 9, pp. 1887-1893, 2003.

[36] I. Cristiani, R. Tediosi, L. Tartara, and V. Degiorgio, "Dispersive waves generation by solitons in microstructured optical fibers," Opt. Express, vol. 12, no. 1, pp. 124-135, 2004.

[37] P. K. A. Wai, H. H. Chen, and Y. C. Lee, "Radiation by solitons at the zero group-dispersion wavelength of single-mode optical fibers," Phys. Rev. Lett., vol. 41, no. 1, pp. 426-439, 1990.

[38] R. Stolen and E. Ippen, "Raman gain in glass optical waveguides," Appl. Phys. Lett., vol. 22, no. 6, pp. 276-278, 1973.

[39] E. A. Golovchenko, P. V. Mamyshev, A. N. Pilipetskii, and E. M. Dianov, "Numerical analysis of the Raman spectrum evolution and soliton pulse generation in single-mode fibers," J. Opt. Soc. Amer. B, vol. 8, no. 8, pp. 1626-1632, 1991.

[40] D. R. Solli, C. Ropers, P. Koonath, and B. jalali, "Optical rogue waves," Nature, vol. 450, no. 13, pp. 1054-1057, 2008.

[41] J. M. Dudley, G. Genty, and B. J. Eggleton, "Harnessing and control of optical rogue waves in supercontinuum generation," Opt. Express, vol. 16, no. 6, pp. 3644-3651, 2008.

[42] J. M. Dudley, G. Genty, and S. Coen, "Supercontinuum generation in photonic crystal fiber," Rev. Mod. Phys., vol. 78, no. 4, pp. 1135-1184, 2006.

[43] R. Trebino, Frequency-Resolved Optical Gating: The Measurement of Ultrashort Laser Pulses. Norwell, MA: Kluwer, 2002.

Laura Abrardi was born in Torino, Italy. She received the M.Sc. degree in nuclear engineering from Politecnico di Torino, Italy, in 2002. Since 2006, she has been working towards the Ph.D. degree at the Institute of Applied Physics, Spanish Council for Research, Madrid, Spain.

From 2003 to 2005, she was with Ecole Polytechnique Fédérale de Lausanne, Lausanne, Switzerland. Her current research interests include nonlinear fiber optics with special regard to supercontinuum generation in optical fibers. 
Sonia Martín-López received the Ph.D. degree from the Universidad Complutense de Madrid, Madrid, Spain, in 2006. The topic of her doctoral dissertation was on experimental and theoretical understanding of continuous-wave pumped supercontinuum generation in optical fibers.

She is currently a Postdoctoral Researcher with the Institute of Applied Physics, Spanish Council for Research, Madrid.

Ana Carrasco-Sanz received the Ph.D. degree from the Universidad de Granada, Granada, Spain, in 2007, with a doctoral dissertation on the synthesis of standard frequencies for WDM system calibration for optical communications.

She is currently with the Department of Optics, Universidad de Granada, Granada, Spain.

Félix Rodríguez-Barrios was born in Zamora, Spain. He received the degree in telecommunications from the Polytechnic University of Madrid, Madrid, Spain, in 2006 and the Master's degree in advanced electronics systems from Carlos III University of Madrid, Leganés, Spain, in 2008. Since 2007, he has been working towards the Ph.D. degree at the Institute of Applied Physics, Spanish Council for Research, Madrid, Spain.

His current research interests include nonlinear fiber optics with special regard to stimulated Brillouin scattering.

Pedro Corredera received the B.Sc. and Ph.D. degrees in physics from the University of Salamanca, Salamanca, Spain, in 1985 and 1989, respectively.

In 1996, he joined the Institute of Applied Physics, CSIC, where he is currently a Research Manager of the Fibre Optics Laboratory. His current research interests include fiber-optic measurements, optical fiber sensors, nonlinear fiber optics, and IR radiometry and detection.

Maria Luisa Hernanz received the B.Sc. degree in physics from the Universidad Autonoma de Madrid, Madrid, Spain, in 1983 and the Ph.D. degree in physics from the Universidad Nacional de Educación a Distancia, Madrid, Spain, in 1989.

In 1984, she joined the Microwaves Group, Institute of Electronics and Communications of Madrid, Spanish Council for Research (CSIC), as a Researcher on satellite antenna communications and microwave networks. In 1996, she joined the Institute of Applied Physics, CSIC, Madrid, where she is currently a Research Member of the Fibre Optics Laboratory. Her research interests include fiber-optic measurements, optical fiber sensors, nonlinear fiber optics, and IR radiometry and detection.

Miguel González-Herráez received the M.Eng. and D.Eng. degrees from the Polytechnic University of Madrid, Madrid, Spain, in 2000 and 2004, respectively.

While working towards the D.Eng. degree, he worked first as a Research Assistant and then a Postdoctoral Fellow in the Applied Physics Institute at the Spanish Council for Research, Madrid, Spain, and had several long stays in the Nanophotonics and Metrology Laboratory, Ecole Polytechnique Federale de Lausanne, Switzerland. In October 2004, he was appointed Assistant Professor in the Department of Electronics, University of Alcalá, Madrid, Spain, where he was promoted to Associate Professor in June 2006. He is the author or coauthor of over 100 papers in international refereed journals and conference contributions and has given several invited talks at international conferences His research interests cover the wide field of nonlinear interactions in optical fibers. 